\title{
APPLICATION OF A PULSED SLOW-POSITRON BEAM TO LOW-DENSITY POLYETHYLENE FILM
}

\author{
E. Hamada ${ }^{a, *}$, N. Oshima ${ }^{b}, \mathrm{~K} \cdot \mathrm{Katoh}^{a}$, T. Suzuki ${ }^{e}, \mathrm{H} \cdot \mathrm{Kobayashi}^{c}$, \\ K. Kondo ${ }^{c}$, I. KanazaWa ${ }^{d}$ And Y. Ito ${ }^{e}$ \\ ${ }^{a}$ Department of Radiological Sciences, Ibaraki Prefectural University of Health Sciences \\ Ami, Ibaraki 300-0394, Japan \\ ${ }^{b}$ Atomic Physics Laboratory, The Institute of Physical and Chemical Research (RIKEN) \\ Wako, Saitama 351-0198, Japan \\ ${ }^{c}$ High Energy Accelerator Research Organization (KEK) \\ Tsukuba, Ibaraki 305-0801, Japan \\ ${ }^{d}$ Department of Physics, Tokyo Gakugei University, Koganei, Tokyo 184-0015, Japan \\ ${ }^{e}$ Research Center for Nuclear Science and Technology, The University of Tokyo \\ Tokai, Ibaraki 319-1106, Japan
}

\begin{abstract}
A pulsing system for a slow-positron beam was applied to study the region near the surface of low-density polyethylene film using positron-annihilation lifetime measurement. The lifetime and intensity of ortho-positronium near the surface were measured as a function of the incident positron energy $(1.6-9.1 \mathrm{keV})$. The size of intermolecular spaces in the surface region $(\sim 1500 \mathrm{~nm})$ was larger than that in the bulk region. This tendency was especially strong in the region around $200 \mathrm{~nm}$ below the surface. On the other hand, the intensity of ortho-positronium decreased at lower incident positron energies, which was attributed to a decrease in the density of the spur electrons and/or an increase in the number of reemitted positrons from the surface.
\end{abstract}

PACS numbers: $78.70 . \mathrm{Bj}$

\section{Introduction}

In polymers, some of the injected positrons form positronium ( $\mathrm{Ps}$ ) with an electron. Ortho-Ps (o-Ps) in which the spins of the two particles have parallel directions, seeks intermolecular spaces and exists inside them until it picks up electrons from the wall of the spaces. There is a semi-empirical relation between the lifetime of $o$-Ps and the size of the spaces [1]. Therefore, a positron-annihilation lifetime (PAL) measurement is useful for investigating the polymer characteristics from the perspective of intermolecular spaces.

* corresponding author 
Positrons emitted from ${ }^{22} \mathrm{Na}$ have a continuous energy distribution from 0 to $540 \mathrm{keV}$. In the conventional measurements, they are directly injected into a specimen, and their mean penetration depth is on the order of $0.1 \mathrm{~mm}$. Thus, only bulk information of the specimen is obtained from the conventional PAL measurement. If we want to study a polymer surface or thin films using a PAL measurement, we need slow positrons with energies of $\sim \mathrm{keV}$. In order to use slow positrons for a PAL measurement, a technique for detecting the injection time of slow positrons into the sample is required. In our group, a system for pulsing slow positrons, which could be constructed on a small scale using a radioisotope (RI), was developed [2].

Recently, it has been observed that the lifetime and intensity of $o$-Ps near the surface of polymers are strongly dependent on the positron incident energy [3-8]. In this paper, we report our preliminary PAL data on the surface region of low-density polyethylene (LDPE) film using our pulsed slow-positron beams.

\section{Experimental}

The sample used in this study was LDPE film, which was kindly supplied by Mitsui Chemical Co. Ltd. The thickness of the sample was $50 \mu \mathrm{m}$; it had a crystallinity of $21.2 \%$, which was determined by X-ray measurements; the density was $0.88 \mathrm{~g} / \mathrm{cm}^{3}$. The sample was used without any pretreatment.

The conventional PAL method was also used to compare the bulk with the surface state of LDPE film. In order to annihilate all positrons within the thickness of the films, twenty films were needed. A positron source, sealed in Kapton foils of $7 \mu \mathrm{m}$ thickness, was sandwiched between two sets of piled sheets made of 20 LDPE films. The details of the conventional PAL method have been presented elsewhere [9].

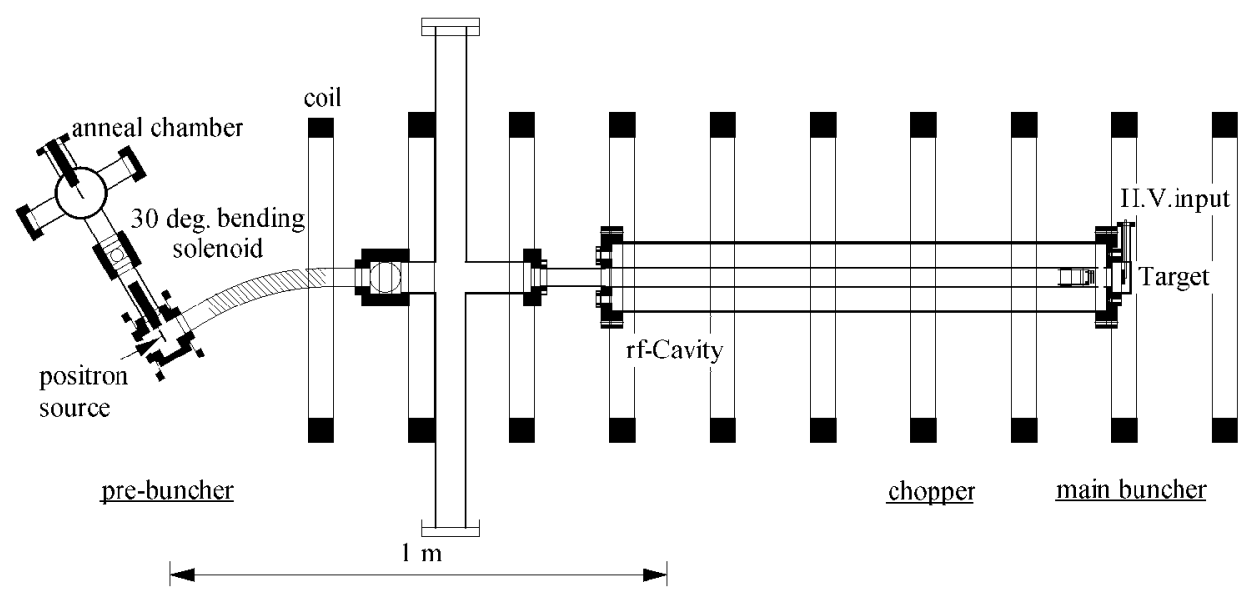

Fig. 1. Layout of the pulsing system for slow positrons. 
The apparatus of the pulsing system is shown in Fig. 1. Slow positrons were produced using ${ }^{22} \mathrm{Na}$ (activity about $740 \mathrm{MBq}$ ) and a tungsten single-crystal moderator $(2 \mu \mathrm{m})$, and magnetically guided to a sample through a vacuum transport line. In order to use these positrons randomly emitted from the moderator for a PAL measurement, positrons were injected into a sample periodically by adjusting the time of flight between the moderator and the sample. Our system comprised a pre-buncher using a time-varying moderator bias method [10], a beam chopper [11] and a main buncher using a radio-frequency (rf) pulsing method [12]. Since this system could achieve a high pulsing efficiency by using an ideal pulsing bias for the pre-buncher, it could be constructed on a small scale by using RI as the positron source. The pulsing period of this system was $40 \mathrm{~ns}$, which was sufficiently longer than the lifetimes of $o$-Ps in polymers. The time resolution, which was defined by a full width at half maximum (FWHM), and the pulsing efficiency were achieved to be about $0.6 \mathrm{~ns}$ and $50 \%$, respectively. Although this time resolution was still larger than that of the conventional PAL measurement system $(\sim 0.25 \mathrm{~ns})$, it can be used to measure the lifetime of $o$-Ps in polymers whose lifetime is about 2 to 3 ns. Our pulsing and PAL measurement system has been detailed elsewhere [2].

\section{Results and discussion}

For the PAL spectrum measured by the conventional method 2 million events were collected at room temperature. The measured spectrum was analyzed with a four-lifetime component by using PATFIT [13]. The lifetimes of para-Ps (0.125 ns) and $o$-Ps (1.1 ns) in the crystalline part were fixed, because an unconstrained analysis with a four-lifetime component was difficult [14]. As a result, the lifetimes and intensities of $o$-Ps in the crystal and the amorphous part were determined to be $(1.1 \mathrm{~ns}, 9.3 \%)$ and $(2.64 \mathrm{~ns}, 21.7 \%)$, respectively.

The PAL spectra by using the pulsed slow-positron beam system were measured as a function of the incident positron energy $(1.6-9.1 \mathrm{keV})$ at room temperature. For each spectrum 1 million events were collected. Assuming one component of the time-resolution function, the measured spectra were fitted using PATFIT and the time resolution was determined to be about $0.6 \mathrm{~ns}$ (FWHM). All the lifetime spectra were analyzed into two lifetime components. The lifetime of the first component was about 1 ns, which was considered to contain the lifetime of para-Ps, free positrons and $o$-Ps in the crystalline part of LDPE. The lifetime of the long-lived component $(2.7 \sim 3.1 \mathrm{~ns})$ roughly agreed with the lifetime of $o$-Ps at the amorphous part in LDPE film.

In Fig. 2, the lifetime and the intensity of the long-lived component are plotted versus the mean implantation depth $(Z)$ of slow positrons, which can be calculated by $Z=(40 / \rho) E^{1.6}$, where $Z$ is expressed in nm, $\rho$ is the density in $\mathrm{g} / \mathrm{cm}^{3}$, and $E$ is the incident energy in $\mathrm{keV}$ [15]. The lifetimes of $o$-Ps at all depths are slightly larger than the value obtained by the conventional PAL method. Because the lifetime of $o$-Ps reflects the size of a space, these results indicate that the size of spaces in the surface region $(\sim 1500 \mathrm{~nm})$ is larger than that in the bulk region. This tendency is especially strong in the region only $200 \mathrm{~nm}$ below the surface. Other research group has observed similar tendencies. For other 


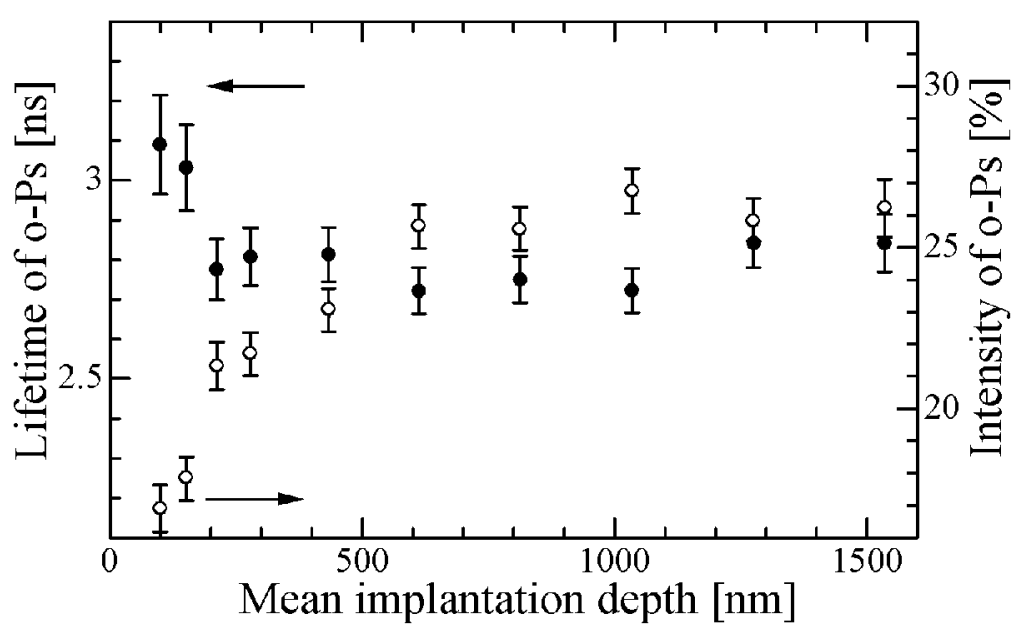

Fig. 2. Lifetime and intensity of $o$-Ps plotted versus the mean implantation depth of slow positrons. The solid and open symbols show the lifetime and intensity of o-Ps, respectively.

LDPE samples (the density is $0.9 \mathrm{~g} / \mathrm{cm}^{3}$ ), Uedono et al. have shown by means of Doppler-broadening measurements and PALS using pulsed slow-positrons that the size of the open spaces in the region between $0 \sim 3 \mu \mathrm{m}$ is larger than in deeper regions [5]. Furthermore, for other polymers, the same results as the above have been reported $[3,4,6,7]$.

On the other hand, the intensity of $o$-Ps increases with increasing mean implantation depth. This phenomenon has been explained as follows. According to the spur model, Ps is formed through a reaction between a thermalized positron and an excess electron created in the terminal positron spur, which is formed when the positron loses the last part of its kinetic energy. It is well known that the terminal positron spur energy, $E_{0}$, is about $0.5 \mathrm{keV} \mathrm{[16].} \mathrm{Recently,} \mathrm{Xie} \mathrm{et} \mathrm{al.} \mathrm{[8]}$ fitted the intensity $\left(I_{o-P s}\right)$ with the incident energy $(E)$ by $I_{o-P s}(E)=I_{o-P s}(0)+$ $\left[I_{o-\operatorname{Ps}}(\infty)-I_{o-\operatorname{Ps}}(0)\right]\left[1-\exp \left(-E / E_{0}\right)\right]$, where $I_{o-\operatorname{Ps}}(0)$ and $I_{o-\operatorname{Ps}}(\infty)$ are the $o$-Ps intensity at $E=0$ and $E=\infty$, respectively. As a result, they obtained $E_{0}$ for several polymers: e.g., $0.74 \mathrm{keV}$ for polycarbonate and $1.4 \mathrm{keV}$ for polystyrene. We also fitted our result with the above equation, and $E_{0}$ for LDPE was determined to be $1.7 \mathrm{keV}$. They have explained this result as follows. If the incident energy of positrons is lower than these large $E_{0}$ values obtained from the above equation, the complete terminal positron spur cannot be formed and the excess electrons in the terminal positron spur can be reduced. Therefore, the intensity of o-Ps decreased with decreasing positron incident energy.

In the case of a surface study using slow positrons, we must consider two effects due to the reemitted Ps and positron from the surface [4]. Since the Ps diffusion length in polymers is very short $[17,18]$, the first effect can be neglected. On the other hand, the second effect cannot be neglected because the diffusion length of positron is much longer than that of Ps. Since the positron diffusion 
constant $\left(D_{e+}\right)$ in polyethylene is $0.8 \mathrm{~cm}^{2} / \mathrm{s}$ [19], the positron diffusion length $L_{e+}=\left(\tau D_{e+}\right)^{1 / 2}$ is estimated to be about $170 \mathrm{~nm}$, where $\tau=0.4 \mathrm{~ns}$ is employed as the lifetime of free positrons. This length is equivalent to the mean implantation depth for $2.4 \mathrm{keV}$.

Thus, it is suggested that the decrease in the o-Ps intensity with decreasing mean implantation depth can be attributed to the decrease in the density of the spur electrons and/or the increase in the number of reemitted positrons from the surface.

\section{Acknowledgments}

The authors would like to thank the Director General, KEK, for special grants. This work was supported in part by a Grand-in-Aid of the Japanese Ministry of Education, Culture, Sports and Science.

\section{References}

[1] S.J. Tao, J. Chem. Phys. 56, 5499 (1972).

[2] E. Hamada, N. Oshima, T. Suzuki, H. Kobayashi, K. Kondo, I. Kanazawa, Y. Ito, Radiat. Phys. Chem. 58, 771 (2000).

[3] A. Uedono, R. Suzuki, T. Ohdaira, T. Mikado, S. Tanigawa, M. Ban, M. Kyoto, T. Uozumi, J. Polym. Sci. B, Polym. Phys. 38, 101 (2000).

[4] H. Cao, J.-P. Yuan, R. Zhang, C.S. Sundar, Y.C. Jean, R. Suzuki, T. Ohdaira, B. Nielsen, Appl. Surf. Sci. 149, 116 (1999).

[5] A. Uedono, R. Suzuki, T. Ohdaira, T. Uozumi, M. Ban, M. Kyoto, S. Tanigawa, T. Mikado, J. Polym. Sci. B, Polym. Phys. 36, 2597 (1998).

[6] H. Cao, R. Zhang, J.-P. Yuan, C.-M. Huang, Y.C. Jean, R. Suzuki, T. Ohdaira, B. Nielsen, J. Phys., Condens. Matter 10, 10429 (1998).

[7] Y.C. Jean, H. Cao, G.H. Dai, R. Suzuki, T. Ohdaira, Y. Kobayashi, K. Hirata, Appl. Surf. Sci. 116, 251 (1997).

[8] L. Xie, G.B. DeMaggio, W.E. Frieze, J. DeVries, D.W. Gidley, H.A. Hristov, A.F. Yee, Phys. Rev. Lett. 74, 4947 (1995).

[9] T. Suzuki, T. Miura, Y. Oki, M. Numajiri, K. Kondo, Y. Ito, Radiat. Phys. Chem. 45, 657 (1995).

[10] E. Hamada, N. Oshima, T. Suzuki, H. Kobayashi, K. Kondo, I. Kanazawa, Y. Ito, Appl. Surf. Sci. 149, 40 (1999).

[11] R. Suzuki, Y. Kobayashi, T. Mikado, H. Ohgaki, M. Chiwaki, T. Yamazaki, T. Tomimasu, Jpn. J. Appl. Phys. 30, L532 (1991).

[12] D. Schödlbauer, P. Sperr, G. Kögel, W. Triftshäuser, Nucl. Instrum. Methods B 34, 258 (1988).

[13] P. Kirkegaard, N.J. Pederson, M. Eldrup, PATFIT-88, Ris $\varnothing-M-2740$, Ris $\emptyset$ National Laboratory, Roskilde (Denmark) 1989.

[14] J. Serna, J.Ch. Abbe, G. Duplatre, Phys. Status Solidi A 115, 389 (1989).

[15] P.J. Schultz, K.G. Lynn, Rev. Mod. Phys. 60, 701 (1988). 
[16] O.E. Mogensen, in: Positron Annihilation in Chemistry, Ed. V.I. Goldanskii, in Springer series in Chemical Physics, Vol. 58, Springer-Verlag, Berlin 1995, p. 66.

[17] E. Hamada, N. Oshima, T. Suzuki, H. Kobayashi, K. Kondo, I. Kanazawa, Y. Ito, in: KEK Proc. 98-4, Eds. S. Sasaki, T. Shibata, H. Takahashi, M. Nakazawa, Tsukuba 1998, p. 172.

[18] Y. Kobayashi, Trends in Macromol. Res. 1, 33 (1994).

[19] W. Zheng, Y. Kobayashi, K. Hirata, T. Suzuki, Radiat. Phys. Chem. 51, 269 (1998). 